\title{
Radiotherapy for Elderly Patients Diagnosed with Laryngeal Carcinoma: A Single Center Experience
}

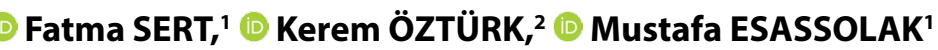 \\ 'Department of Radiation Oncology, Ege University Faculty of Medicine, İzmir-Turkey \\ ${ }^{2}$ Department of Otolaryngology, Ege University Faculty of Medicine, İzmir-Turkey
}

\begin{abstract}
OBJECTIVE
To evaluate the efficacy and toxicity of definitive and postoperative radiotherapy (RT) for laryngeal carcinoma patients aged $\geq 70$ years.

\section{METHODS}

Data of patients between December 2006 and January 2016 were retrospectively analyzed. In total, 66 (4 females and 62 males) patients were evaluated for this analysis. The median age was 74 (range, 70-86 years) years. The total RT dose was $70 \mathrm{~Gy}$ and 60-66 Gy for definitive and postoperative settings with 2 Gy per fraction, respectively.

\section{RESULTS}

The median follow-up time was 29.5 (range, 4-126 months) months. Using the tumor, node, metastasis (TNM) classification, tumors were stage IIA (18.2\%), stage IIIA (31.8\%), stage IIIB (3\%), and stage IVA (47\%). The purpose of RT was definitive for 33 (50\%) patients and postoperative for 33 (50\%) patients. Concomitant chemotherapy was used in 21 (31\%) patients with $9(42 \%)$ receiving cetuximab and 12 (58\%) receiving cisplatin. Cisplatin dose reduction was needed in $20 \%$ patients, but cetuximab was used as per a suggested protocol. The 3-year local relapse free (LRF), metastases free (MF), and overall survival rates for all patients were $74 \%, 84 \%$, and $48 \%$, respectively. Only 26 (40\%) patients died due to disease specific problems, and 18 (27\%) patients died of unknown causes. In the toxicity evaluation, no grade 3-4 adverse effect was seen. Any type of cytopenia status was reported in 14 (21\%) patients, and this rate was statistically higher in concomitant radiochemotherapy group $(p=003)$.
\end{abstract}

\section{CONCLUSION}

RT seems to be an effective therapy in elderly population either alone or concomitant with chemotherapy in definitive and postoperative settings. In contrast, comorbid diseases should be managed carefully in the treatment and follow-up periods.

Keywords: Cisplatin; cetuximab; elderly; laryngeal carcinoma; radiotherapy.

Copyright $\odot$ 2018, Turkish Society for Radiation Oncology

\section{Introduction}

Elderly patients are potentially sensitive to cancer treatments due to comorbidities and/or geriatric syndromes. By 2050 , more than $25 \%$ of the US population and more than $30 \%$ of the European population will be over 60 years of age.[1] Nearly $50 \%$ of all cancers are seen in the population older than 65 years.[2] When we consider the ages between 70 and 75 years, head and neck cancers account $6 \%-32 \%$ of all cancer diagnosis.[3] Approxi- 
mately 45.000 new head and neck squamous cell carcinomas (SCCs) are diagnosed annually in the US, with at least one-quarter seen in patients older than 70 years. $[4,5]$ In Turkey, the numbers are not clear owing to the lack of cancer registrations. However, it is known that cancer diagnosis in the elderly population is increasing in our country as well. Laryngeal carcinoma is the most commonly seen head and neck cancer type in Turkey.

Tailoring cancer therapy and applying required intensive treatments for older patients with laryngeal carcinoma can be challenging. Geriatric patients are generally excluded from clinical trials; current data were established with results of those trials, and hence evidence-based data involving geriatric population are limited. Concomitant radiochemotherapy (RCT) is accepted as standard for organ preservation in the curing stage III/IV disease and for adjuvant treatment in high risk operated patients.[6] Age is not a contraindication for both postoperative and concomitant treatment. A comprehensive risk evaluation must be performed for reducing the morbidity and mortality in the older group of patients. There are different approaches to be considered in case of the treatment of elderly patients. Cetuximab is an epidermal growth factor receptor inhibitor used for the treatment of metastatic colorectal cancer, metastatic non-small cell lung cancer, and head and neck cancer. Cetuximab is a chimeric (mouse/human) monoclonal antibody given by intravenous infusion. Concomitant radiotherapy (RT) and cetuximab association has shown superior results to RT alone for head and neck cancers, with a $13 \%$ absolute improvement in locoregional control and a $10 \%$ absolute improvement in the 3-year survival, with a minimal increase in toxicity.[7] This agent is an option for the elderly population for concomitant usage rather than RT alone and/or cisplatin.

Data are insufficient in terms of providing a precise prognosis for elderly patients diagnosed with laryngeal carcinoma despite its rising incidence. The purpose of this retrospective analysis was to evaluate the efficacy and toxicity of definitive concomitant RCT or postoperative RCT/RT for patients aged $\geq 70$ years with supraglottic laryngeal carcinoma.

\section{Materials and Methods}

A retrospective single center data evaluation was performed in the Radiation Oncology Department of Ege University Medical School \& Hospital in Turkey. Turkey is the one of endemic countries for laryngeal carcinoma. Approval from the research ethics board was obtained from Ege University Ethic Committee with the decision number 16-5.1/9. Patient files were evaluated retrospectively. Inclusion criteria were age $\geq 70$ years at the time of diagnosis, histologically proven laryngeal SCC located in the supraglottic area, and treated with definitive or postoperative RT. Exclusion criteria were early stage disease or previous RT and metastases at the time of diagnosis. A concomitant chemotherapeutic agent was selected according to the stage of disease and the performance status of the patient. Tumors were staged using the tumor, node, metastasis (TNM) classification (seventh edition, 2009).[8] Toxicity evaluation was performed weekly during RT using the National Cancer Institute Common Toxicity Criteria, v3, and then patients were followed at regular intervals.[9]

A simulation computed tomography (CT) scan was performed with a custom thermoplastic mask in the treatment position with slices of $3 \mathrm{~mm}$. The gross tumor volume (GTV) was determined as a clinically visible tumor in a clinical examination, endoscopy, CT scan, magnetic resonance imaging, and positron emission tomography scan. The clinical target volume (CTV) was determined according to the experiences regarding the risks of microscopic disease around the GTV. A planning target volume was created by giving an additional $0.5 \mathrm{~cm}$ margin to CTV (for IMRT plans, preferred to be given $0.3 \mathrm{~cm}$ ).

Treatment was delivered using an Electa linear accelerator. A minimum 3-dimensional conformal RT technique was used. For definitive purposes, in total, 66-70 Gy with 2 Gy per fraction to the primary tumor and involved nodes and between 50 Gy and 56 Gy to the bilateral uninvolved cervical lymph nodes were used. For postoperative purposes, in total, 60-66 Gy with 2 Gy per fraction to the microscopic suspicious areas was used.

Cisplatin was delivered weekly $40 \mathrm{mg}$ per square meter during the course of RT. In case of renal failure and comorbid problems, cetuximab was preferred. Cetuximab was delivered by starting 1 week before RT at a loading dose of $400 \mathrm{mg}$ per square meter infusion, followed by weekly $250 \mathrm{mg}$ per square meter infusion during the course of RT.

A statistical analysis was performed using the Statistical Package for Social Sciences, version 18 (IBM, US) software. Survival and recurrence outcomes were calculated from the day when the cancer diagnosis was proven. Local control (LC), regional control (RC), disease specific survival, and overall survival (OS) rates were analyzed using the Kaplan-Meier curves. Log rank and Cox regression analysis were performed for detecting prognostic factors. 


\section{Results}

Sixty-six patients were included between 2007 and 2016. The median follow-up period was 29.5 (range, 4-126 months) months. The median age was 74 (range, 70-86 years) years. The Median Karnofsky Performance Status was $\geq 80 \%$. Four (6\%) patients were females, and $62(94 \%)$ patients were males. The included patients were questioned in terms of comorbid diseases determined having a diagnosis of any additional disease required medical therapy. Thirty (45\%) patients did not have any comorbid disease; 36 (55\%) patients had minimum one comorbid disease. The patient characteristics are shown in Table 1.

Table 1 Patient Characteristics

\begin{tabular}{|c|c|}
\hline Characteristic & Number (\%) \\
\hline Median Age & $74(70-86)$ years \\
\hline \multicolumn{2}{|l|}{ Sex } \\
\hline Male & $62(94 \%)$ \\
\hline Female & $4(6 \%)$ \\
\hline \multicolumn{2}{|c|}{ Karnofsky Performance Status } \\
\hline$\geq 80 \%$ & $56(85 \%)$ \\
\hline$<80 \%$ & $10(15 \%)$ \\
\hline \multicolumn{2}{|l|}{ Tumor Category } \\
\hline $\mathrm{T} 1$ & $1(1.5 \%)$ \\
\hline $\mathrm{T} 2$ & $20(30.3 \%)$ \\
\hline T3 & $24(36.4 \%)$ \\
\hline $\mathrm{T} 4 \mathrm{a}$ & $21(31.8 \%)$ \\
\hline \multicolumn{2}{|l|}{ Nodal Category } \\
\hline No & $44(66.7 \%)$ \\
\hline N1 & $6(9.1 \%)$ \\
\hline $\mathrm{N} 2 \mathrm{a}$ & $9(13.6 \%)$ \\
\hline $\mathrm{N} 2 \mathrm{~b}$ & $4(6.1 \%)$ \\
\hline $\mathrm{N} 2 \mathrm{C}$ & $3(4.3 \%)$ \\
\hline \multicolumn{2}{|l|}{ AJCC Stage } \\
\hline IIA & $12(18.2 \%)$ \\
\hline IIIA & $21(31.8 \%)$ \\
\hline IIIB & $2(3.0 \%)$ \\
\hline IV & $31(47.0 \%)$ \\
\hline \multicolumn{2}{|l|}{ Surgery } \\
\hline+ & $33(50 \%)$ \\
\hline- & $33(50 \%)$ \\
\hline \multicolumn{2}{|l|}{ Chemotherapy } \\
\hline+ & $21(32 \%)$ \\
\hline- & $45(68 \%)$ \\
\hline Chemotherapy Agent & $21(32 \%)$ \\
\hline Cisplatin & $9(13.6 \%)$ \\
\hline Cetuximab & $12(18.2 \%)$ \\
\hline \multicolumn{2}{|l|}{ Comorbid Disease } \\
\hline+ & 40 (60.6\%) \\
\hline- & $15(22.7 \%)$ \\
\hline Unknown & $11(16.6 \%)$ \\
\hline
\end{tabular}

Using the TNM classification, tumors were T1 in 1 (1.5\%), T2 in $20(30.3 \%), \mathrm{T} 3$ in $24(36.4 \%)$, and T4a in $21(31.8 \%)$ and N0 in $44(66.7 \%), \mathrm{N} 1$ in $6(9.1 \%), \mathrm{N} 2 \mathrm{a}$ in $9(13.6 \%), \mathrm{N} 2 \mathrm{~b}$ in $4(6.1 \%)$, and $\mathrm{N} 2 \mathrm{c}$ in $3(4.5 \%)$ patients. RT was used in one-half of the patients for definitive purposes and in the other half for postoperative purposes. The total RT dose was $70 \mathrm{~Gy}$ and $60-66 \mathrm{~Gy}$ for definitive and postoperative settings, respectively, with 2 Gy per fraction. Fifty-five (83.3\%) patients were treated with the conventional 3D RT, and $11(16.7 \%)$ patients were treated with intensity modulated RT. RT was interrupted due to the acute toxicities of treatment in 94\% of patients. The median overall radiation treatment time was 48 (range, 31-78 days) days. The median duration of treatment breaks was 7 (range, 3-12, days) days.

Concomitant chemotherapy was used in 21 (31\%) patients and 9 (42\%) of those received cetuximab and 12 (58\%) cisplatin. Cisplatin dose reduction was needed in $20 \%$ patients due to weight loss and/or renal functional problems. Cetuximab was used with a suggested protocol, but some dose modifications were required. Four patients had an interruption of cetuximab therapy.

Three-year local relapse free (LRF), metastases free (MF), and OS rates for all patients were $74 \%, 84 \%$, and $48 \%$, respectively, (Figure 1a, 1b, and 1c). The median survival periods were 28 months for LRF, 31 months for $\mathrm{MF}$, and 30 months for OS. For concomitant CT group, the 3 year LRF, MF, and OS rates were $55 \%, 74 \%$, and $36 \%$, respectively. Only $26(39.4 \%)$ patients died due to

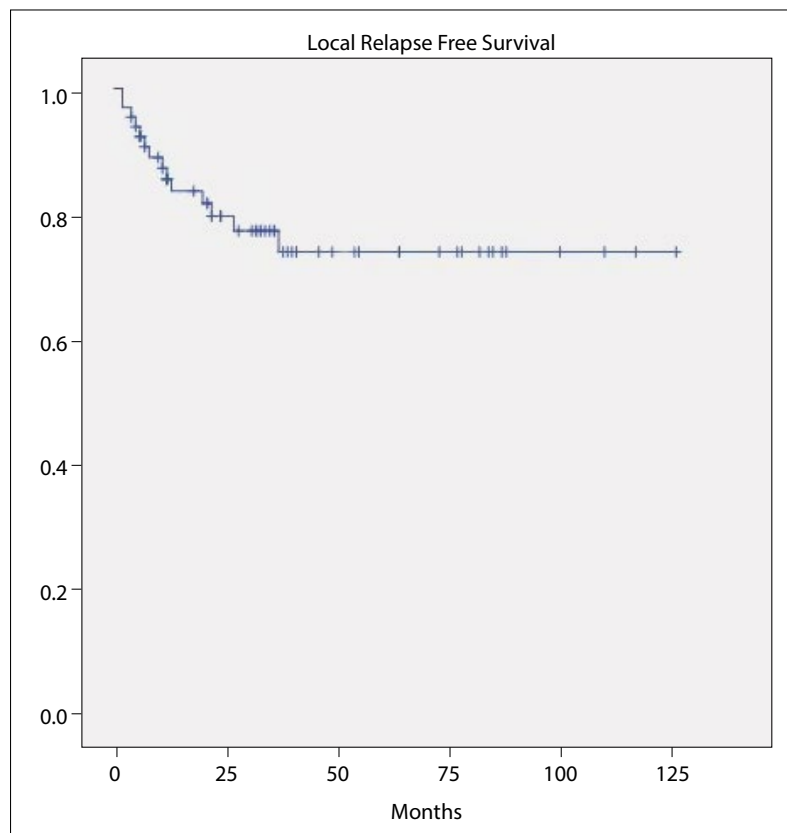

Fig. 1a. Local relapse free survival curve for all patients. 


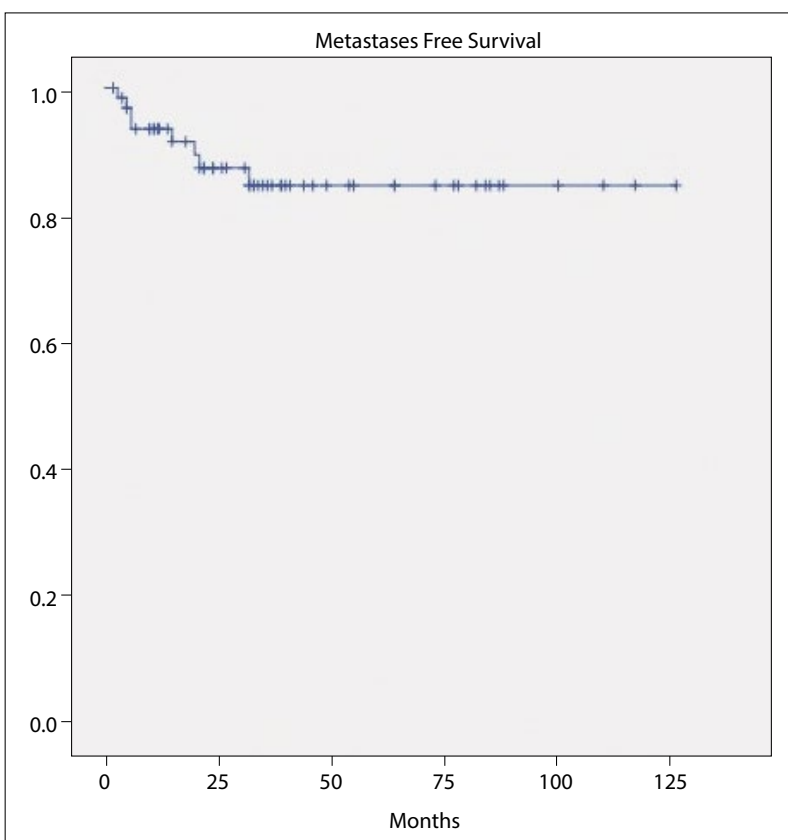

Fig. 1b. Metastases free survival curve for all patients.

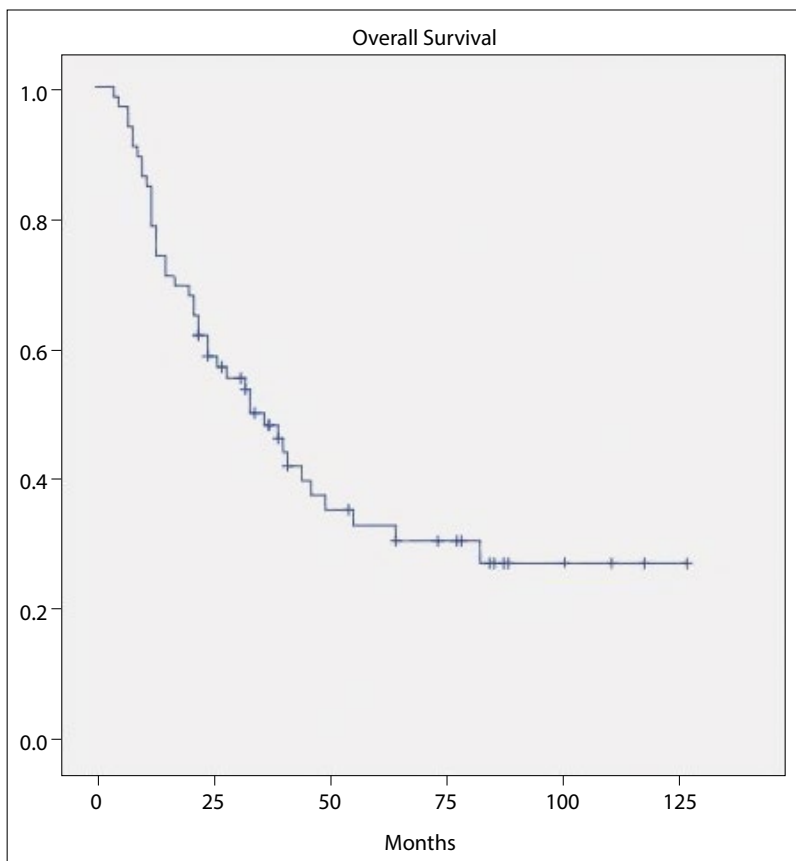

Fig. 1c. Overall survival curve for all patients.

disease specific problems, and 18 (27.2\%) patients died of unknown causes.

We compared the treatment outcomes in terms of treatment purposes (definitive vs postoperative). In the log-rank evaluation, the estimated OS times were 44 and 63 months for definitive and postoperative treated pa-
Table 2 Toxicity Results

\begin{tabular}{lc} 
Type & Number (\%) \\
\hline Cytopenia & \\
Grade II & $14(21 \%)$ \\
- & $52(59 \%)$ \\
Nausea & \\
- & $61(92.4 \%)$ \\
Grade I & $4(6.1 \%)$ \\
Grade II & $1(1.5 \%)$ \\
Vomiting & \\
- & $61(92.4 \%)$ \\
Grade I & $3(4.5 \%)$ \\
Grade II & $2(1.5 \%)$ \\
Esophagitis & \\
- & $52(79 \%)$ \\
Grade I & $10(15 \%)$ \\
Grade II & $4(6 \%)$ \\
Fatigue & \\
+ & $9(13.6 \%)$ \\
- & $57(86.4 \%)$ \\
Radiation Pneumonitis & \\
+ & $21(32 \%)$ \\
- & $45(68 \%)$ \\
\hline
\end{tabular}

tients, respectively $(\mathrm{p}=058)$. There was a favorable trend toward the postoperative group. A similar comparison was done for the LRF time, and the estimated LRF times were 97 and 110 months for the definitive and postoperative treated patients, respectively $(\mathrm{p}=460)$.

Eighty-five percent of the patients had nutritional supports. Oral nutritional supplements alone were sufficed for $52 \%$ patients but $46 \%$ needed enteral nutrition and $2 \%$ parenteral nutrition. Nasogastric tubes or percutaneous enteral gastrostomy (if necessary) was used for parenteral nutrition. Percutaneous enteral gastrostomy was planned to close within 1-2 months after treatment and nasogastric tubes were removed approximately 2 weeks after treatment.

In the toxicity evaluation, no grade 3-4 adverse effect was seen. Skin rash due to cetuximab was seen in nearly all the included patients, but a treatment break was needed for only one patient due to rush. All hematologic and non-hematologic adverse effects were seen higher in the concomitant therapy group but none of them, except cytopenia, was found to be significant in the statistical analyses. Any grade II cytopenia status was reported in $14(21 \%)$ patients, and this rate was statistically significant in the concomitant RCT group $(\mathrm{p}=003)$. The details of the toxicity results are shown in Table 2. There was no statistically significant difference in terms 
of grade II cytopenia, fatigue, esophagitis, nausea, and vomiting between the patients treated with definitive or postoperative purposes (all $\mathrm{p}$ values were $>.05$ ). In contrast, grade I/II rush was statistically significant for definitively treated patients $(\mathrm{p}=004)$ and grade I/II rush was statistically significant for postoperatively treated patients $(\mathrm{p}=044)$.

\section{Discussion}

The current guidelines suggest that potential curative treatments should be recommended to patients independent of age for nearly all head and neck cancers. [10] Cisplatin is the major chemotherapeutic agent for concomitant usage, but cetuximab can be considered an alternative agent for patients who are not suitable for cisplatin.[11] Patient compliance and management of treatment toxicities can be difficult when planning to use RT concomitant with cisplatin or cetuximab.[11] This group of patients should be accepted as a special cohort in terms of both RT and concomitant chemotherapy.

In a national cancer database analysis done by Ward MC. et al, elderly patients with head and neck cancer treated with definitive RT were evaluated in terms of treatment results and toxicity outcomes.[12] They included elderly patients treated between 2004 and 2012 $(n=4165)$. The median follow-up period was 26 months (range, $1.8-125$ months), and $80.4 \%$ of the patients received systemic therapy. The 3 -year OS rate was $51.6 \%$, and the use of systemic therapy was found to be associated with improved OS in a multivariate analysis $(\mathrm{p}<.0001)$. During the present study, an increase in the frequency of systemic therapy use from $64 \%$ in 2004 to $86 \%$ in 2012 was noted.[12] In the evaluation of our patient data, we found that the 3-year OS was $48 \%$ for all cohorts, and this result was consistent with Ward MC. et al. However, the OS of the concomitant CT group in our data was lower compared to the Ward MC. et al study. This could be a result of included patients' diagnosis. They included whole head and neck cancer patients, but our results were focused only on laryngeal cancer patients. Surgical approaches were encouraged in our cohort, and hence implementation difficulties for systemic agents occurred in our treatment group.

In other cancer database analysis performed by Amini A. et al, elderly patients with locally advanced head and neck cancer were evaluated in this analysis. [13] They evaluated the outcomes of used concurrent CRT in this group of patients. For this purpose, they included 4042 patients and $63 \%(n=2538)$ of them received concurrent
CRT. The median follow-up period was 19 months. In addition, they showed survival benefit with CRT for the patients over 70 years of age. They concluded that patients older than 70 years should not be denied concurrent CRT solely based on age; additional factors, including the performance status and the tumor stage, should be considered.[13] In our examined group, a significant LC difference was seen between the two groups. The patients with concurrent CRT had better LC rates than did those without concurrent CRT ( $\mathrm{p}=019$ ). This shows similarity with literature. If patients' performance status and biochemical functional tests are appropriate for chemotherapy, a concurrent chemotherapeutic agent should be for this patient independent of the patient's age.

In an Italian single centered study, the data of elderly patients used concomitant RT and cetuximab was published in 2017.[14] Falk AT et al. presented a 4 year experience regarding concomitant cetuximab and RT in elderly patients. They evaluated 35 patients' with a median follow-up of 22 months. The included tumor sites were mostly oropharynx (57.1\%), larynx (20\%), and hypopharynx (14.3\%). The median RT dose was 70 (range, 60-70) Gy, and the purpose of RT was mostly definitive treatment. The 2-year local-regional relapse and metastatic relapse-free survival rates were $59 \%$ and $74 \%$, respectively. They concluded that concomitant RT and cetuximab seem to be an effective therapy for elderly patients.[14] Surgical approaches are believed to be the first treatment option for this group of patients. Definitive and adjuvant RT are recommended for mainly complicated patients with comorbidities. Therefore, the concomitant chemotherapy patient number was 21 (31.8\%), with cisplatin used in $12(18.2 \%)$ and cetuximab in $9(13.6 \%)$ patients. In the toxicity analysis, we did not detect any grade 3-4 toxicity adverse effect during the treatment period. In contrast, the 2-year LRF survival and OS rates were lower compared to the Falk AT et al. trial (55\% and 38\%). This could be a result of referred patients' comorbidities avoiding surgery and the nature of laryngeal cancer.

Patient compliance seemed acceptable with all evaluated patients completing treatment without dose reduction in the cetuximab cohort, and only $20 \%$ of patients needed a dose reduction in the cisplatin cohort. However, RT was interrupted due to the acute toxicities of treatment in $94 \%$ of patients. A review by Fowler JF et al showed a median value of $14 \%$ of LC rate loss per week of extra overall treatment time.[15] In our data, all patients completed treatment in the planned treatment time and patients were not compensated for unplanned interruptions. 
Currently, published data involving RT for the elderly patients with laryngeal cancer are scarce. Available data mostly include all the primary sites of head and neck region for these elderly patients. This renders complexity when deciding for the elderly patients with laryngeal cancer. $[16,17]$ Used concomitant chemotherapeutic agents and the conditions of usages are also not homogeneous. Our series represents a group of elderly patients with laryngeal cancer, which is the most commonly seen head and neck cancer in Turkey, who were treated with $\mathrm{RT}+/$ - concomitant chemotherapy. When we compare the results with the best supportive care, applied RT with or without chemotherapy shows promising treatment outcomes for the elderly patients.

\section{Conclusion}

RT with or without chemotherapy seems to be effective for elderly patients diagnosed with laryngeal carcinomas. Our results show similarity with literature concerning its efficacy and toxicity when compared with younger patients. Surgical approaches may be considered according to the indications for laryngeal cancer treatment independent of the patient's age. Age is not a contraindication for both surgery and RT with or without chemotherapy. In addition, comorbid diseases should be managed carefully in both treatment and follow-up periods.

Peer-review: Externally peer-reviewed.

Conflict of Interest: None declared.

Ethics Committee Approval: Approval from the research ethics board was obtained from Ege University Ethic Committee with the decision number 16-5.1/9.

Financial Support: None declared.

Authorship contributions: Concept - F.S.; Design - F.S., K.Ö.; Supervision - M.E.; Data collection \&/or processing F.S., K.Ö., M.E.; Analysis and/or interpretation - F.S., M.E.; Literature search - F.S.; Writing - F.S.; Critical review - K.Ö., M.E.

\section{References}

1. United Nation-Population-Departments of Ecomonomic and Social Affairs-Population AGEING 2002. Available at: http://www.un.org/esa/population/publications/ageing/Graph.pdf. Accessed Dec 22, 2017.

2. Balducci L, Beghe' C. Cancer and age in the USA. Crit Rev Oncol Hematol 2001;37(2):137-45.

3. Bernardi D, Barzan L, Franchin G, Cinelli R, Balestreri $\mathrm{L}$, Tirelli U, et al. Treatment of head and neck cancer in elderly patients: state of the art and guidelines. Crit Rev Oncol Hematol 2005;53(1):71-80.
4. Siegel RL, Miller KD, Jemal A. Cancer statistics, 2015. CA Cancer J Clin 2015;65(1):5-29.

5. Muir CS, Fraumeni JF Jr, Doll R. The interpretation of time trends. Cancer Surv 1994;19-20:5-21.

6. Adelstein DJ, Li Y, Adams GL, Wagner H Jr, Kish JA, Ensley JF, et al. An intergroup phase III comparison of standard radiation therapy and two schedules of concurrent chemoradiotherapy in patients with unresectable squamous cell head and neck cancer. J Clin Oncol 2003;21(1):92-8.

7. Bonner JA, Harari PM, Giralt J, Azarnia N, Shin DM, Cohen RB, et al. Radiotherapy plus cetuximab for squamous-cell carcinoma of the head and neck. $\mathrm{N}$ Engl J Med 2006;354(6):567-78.

8. Sobin LH, Gospodarowicz MK, Wittekind C. TNM Classification of malignant tumors. 7th ed. Available at: http://eu.wiley.com/WileyCDA/WileyTitle/productCd-1444332414.htlm. Accessed Dec 22, 2017.

9. Protocol Development. CTEP. Available at: http://ctep. cancer.gov/protocolDevelopment/electronic_applications/ctc.htlm. Accessed Dec 22, 2017.

10. Sarris EG, Harrington KJ, Saif MW, Syrigos KN. Multimodal treatment strategies for elderly patients with head and neck cancer. Cancer Treat Rev 2014;40(3):465-75.

11. Magrini SM, Buglione M, Corvò R, Pirtoli L, Paiar F, Ponticelli $\mathrm{P}$, et al. Cetuximab and Radiotherapy Versus Cisplatin and Radiotherapy for Locally Advanced Head and Neck Cancer: A Randomized Phase II Trial. J Clin Oncol 2016;34(5):427-35.

12. Ward MC, Reddy CA, Adelstein DJ, Koyfman SA. Use of systemic therapy with definitive radiotherapy for elderly patients with head and neck cancer: A National Cancer Data Base analysis. Cancer 2016 Aug 9 [Epub ahead of print], doi: 10.1002/cncr.30214.

13. Amini A, Jones BL, McDermott JD, Serracino HS, Jimeno A, Raben D, et al. Survival outcomes with concurrent chemoradiation for elderly patients with locally advanced head and neck cancer according to the $\mathrm{Na}$ tional Cancer Data Base. Cancer 2016;122(10):1533-43.

14. Falk AT, Hébert C, Tran A, Chand ME, Leysalle A, Thariat J, et al. Radiotherapy for elderly patients and cetuximab, a monocentric study. Eur Arch Otorhinolaryngol 2017;274(2):1061-5.

15. Fowler JF, Lindstrom MJ. Loss of local control with prolongation in radiotherapy. Int J Radiat Oncol Biol Phys 1992;23(2):457-67.

16. Ho C. Cetuximab in locally advanced head-andneck cancer: defining the population. Curr Oncol 2010;17(4):48-51.

17. Kataria T, Gupta D, Bisht SS, Goyal S, Basu T, Srivastava A, et al. Chemoradiation in elderly patients with head and neck cancers: a single institution experience. Am J Otolaryngol 2015;36(2):117-21. 